\title{
Technische Neuheiten aus den Gebieten der Medizin, Oeffentlichen Gesundheitspflege und Krankenpflege.
}

Von Dr. M. Schall in Halensee.

Die Automobilfabrik'Turicum, A.-(t. in Uster ${ }_{\alpha}$ (Schweiz), ließ sich unter D. R. P. 225030 eine Trag- und Fahrbahre mit zwei aus scharnierartig verbundenen Finzelteilen zusammengesetzten Holmen schützen. Das Neue dieser Einrichtung besteht darin, daß alle Teile der Holme nach derselben Richtung klappbar und feststellbar sind und nach $\mathrm{U}_{\mathrm{m}}$. klappung in dieser Richtung und Feststellung der Scharniere 4, 5, 6, 7 zur Bildung eines Tragkastens dienen (vgl. Fig. 1). Die gebogenen Holmenden sind zur Bildung von Schlittenkufen in entgegengesetzter Richtung umklappbar (vgl. Fig. 2).

Fig. 1.
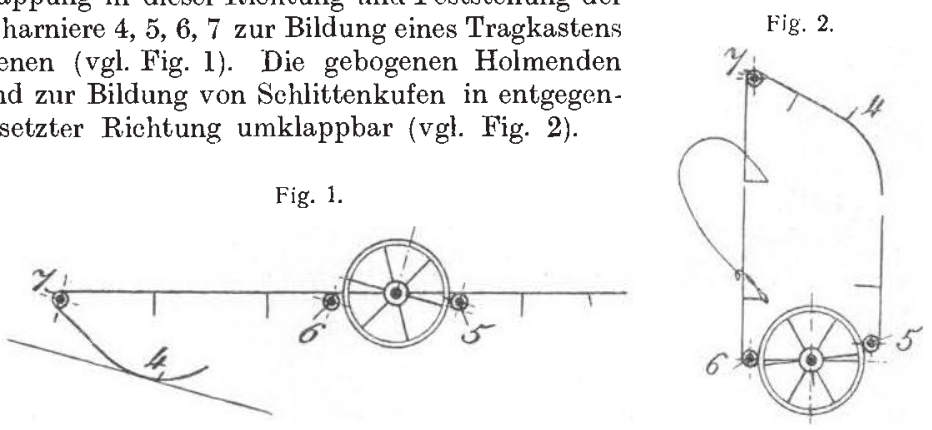

Eine zusammenlegbare Tragbalire mit von Kniegelenken gebildeten Stiitzen bildet den Gegenstand des D. R. P. 225015 (K. Herholdt in Brandenburg a. H. und Mannesinannröliren-Werke in Düsseldorf). Von den bekannten Bahren dieser Art unterscheidet sich die neue Tragbahr? dadureh, daß die nit Hilfe verschiebharer Hïlsen $b^{3}, b^{4}$ (vgl. Fig. 3)

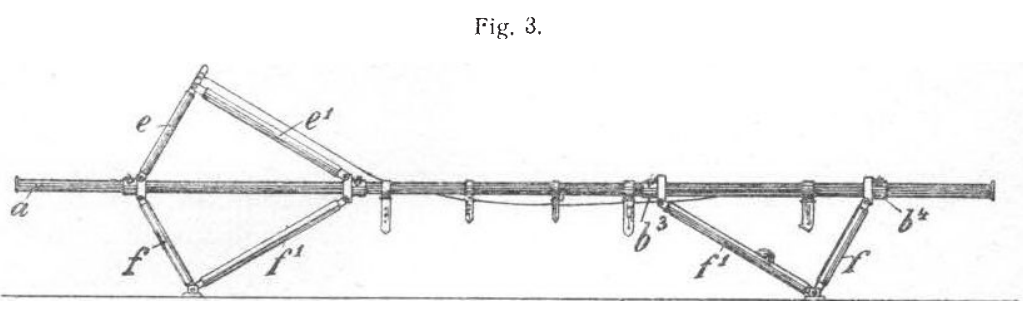

auf den Holmen a feststellbaren beiden Schenkel der Kniegelenke e, $e^{1}, f, f^{1}$ durch Auflager verbunden sind und als $F \ddot{1} ß e$ dienen und durch den Druck der Last in der gestreckten Lage erhalten werden. Gemäß der Erfindung ist ferner auch die Kopfstiitze aus Kniegelenken gebildet. Falls die Bahre in zusammengelegtem Zustand eine geringere Länge betsitzen soll, können die Holme mit Scharnieren versehen werden, un sie in der Längsrichtung zusammenklappen zu können, wobei dann dürch eine mittlere Strebe die erforderliche Steifigkeit zu erzielen wäre. Gegebenenfalls könnte man die Holme auch aus ineinanderschiebbaren Rohren herstellen, beispielsweise auch die Handgriffe einschiebbar machen.

Um einen vierrädrigen Wagen mit an Vordergestell befestigtem und in einer am Hintergestell angebrachten Hülse gelagertem Langbaum zum Verwundetentransport in mebenem Gelände handelt es sich bei D. R. P. 225029 (Marie Henri Ch. Piusais in Montargis, Frk.). Der' langlaum K (vgl. Fig. 4) ist (lrehbar und gegen Herausziehen gesichert

Fig. 4.

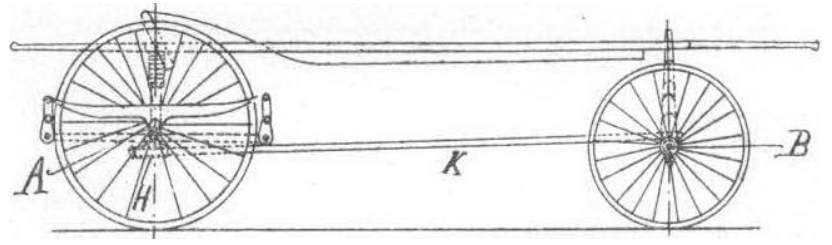

in der Hülse $\mathrm{H}$ gelagert, oder ein hohler Langbaum ist drehbar und gexen Herausziehen gesichert un einen am Hintergestell A angeordneten Zapfen gelagert, sodaß sich clas Hintergestell frei um das Fnde des Langbaums beim Neigen des Vordergestells B drehen kann und dits Kippen des Wagens in unebenem crelände vermieden wird. 
Zwei neve Konstruktionen von Krankenbetten lernen wir aus den D. R. Patenten No. 225410 und 225411 kennen.

Ersteres betrifft ein zusammenlegbares Krankenbett mit senkrecht stellbarer Matratze und gegen diesc anlegbaren Stirnwänden. Die bekannten Betten dieser Art müssen im zusammengefalteten Zustand
Fig. 5.

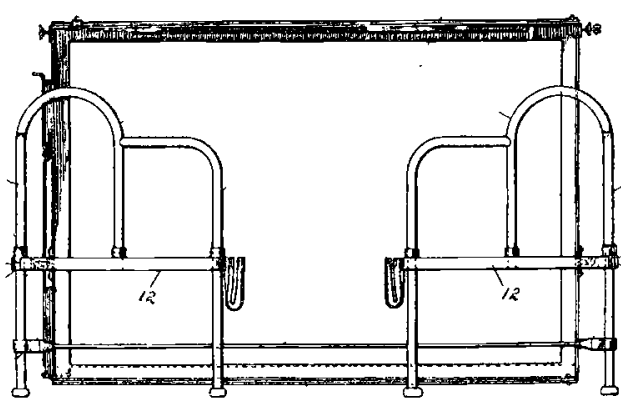
gegen die Wand oder dergleichen angelegt werden, da sie andernfalls umfallen würden. Dieser Uebelstand wird bei der Bettstelle gemäß der Frfindung dadureh vermieden, daß die Stirnwände (vgl. Fig. 5) aus zwei gelenkig nitcinander vorbundenen Teilon bestelien und nur ein Teil der Kopf. und Fußstirnwand gegen die Matratze angelegt wird, während der übrige Teil der Kopf- und F'ußstirnwand in seiner Stellung verbleibt und als Stütze für die zusammengefaltete Bettstclle dient, sodaß cin weiterer Stützpunkt für die Bettstclle nicht inehr erforderlich ist.

Un ein Krankenbett, das den Kranken in jede Lage zu bringen gestattet, ohne ihn ganz aus dem Bett entfernen zu niissen, handelt es sich bei D. R. P. 225411 (W. Müller in Hallc a. S.). 'Der inittels Rollen g Fig 6.

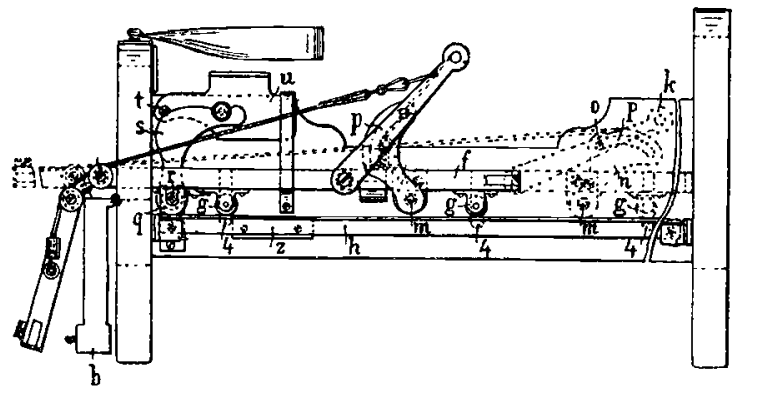

auf Schicnen h glcitende Matratzenrahmen f kann ïber die herabgeklappte Fußwand $\mathbf{b}$ des Bettgestclls herausgezogen werden (vgl. Fig. 6); der herausragende Tcil des Rahmens läßt sich alsdann durch Hochstellen der Kopfstütze $K$ senken.

Ein Trepan ist durch D. R. P. 224031 geschützt (Th. J. Fr. M. de Martel de Janville in Paris). Er läßt die Anwendung zylindrischer Bohrer und ein Bohren ohne Unterbrechung $z u$, sodaß das $A b$ -

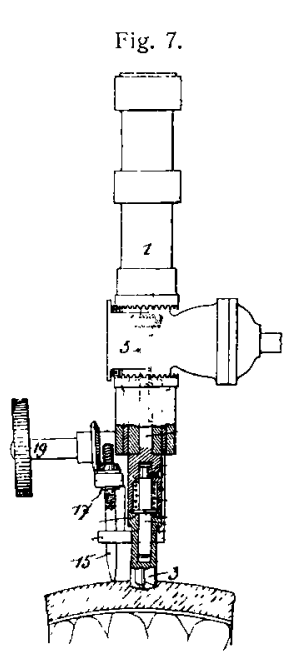
heben des Bohrers, das Untersuchen des Fort. schreitens des Bohrens und eine besondere Geschicklichkeit unnötig werden und keine Gefahr der Verletzung der Hirnhaut besteht. Der Bohrer ist mit seincr Antriebsvorrichtung durch cine unter der Einwirkung einer Feder stehende Kupplung verbunden, welche bei einem in der Achse des Bohrers wirkenden Druck diesen selbsttätig mit der Antriebsvorrichtung so lange verbindet, als der anzubohrende Knochen dem Clegendruck Widcrstand leistet, und die selbsttätig gelöst wird, sobald die Knochenwand dem Bohrdrucke nachgibt. Das Eindringen des Bohrers in den Knochen wird durch eine regelbare Stützvorrichtung begrenzt, welche sich gegen den anzubohrenden Knochen legt. Die Stützvorrichtung besteht "aus einem seitlich vom Bohrer 3 in einem ${ }^{\prime r}$ am Körper 1 befestigten Winkelstïck verschiebbaren Bolzen 15 , der mittels einer Stellvorrichtung 17, 19 in der Längsrichtung des Bohrers eingestellt wird, sodaB sein freies, gegen den Knochen gerichtetes Ende eine bestimnite Höhenlage zur Unterkante des Bolırers cinnehmen kann (vgl. Fig. 7).

Gegenstand des Patentes No. 225031 ist eine in einem Halter beIestigte Sonde (J. Beutelrock \& Sohn in München). Die Sonde wird durch den Halter geführt und ist in ihn so gelagert, daß sie an den der Sondenspitze gegenüberliegenden Fnde des Halters herausragt (vgl. Fig. 8) und an diesem Ende für verschiedene Stichtiefen cingestellt werden kann. Das untere. Fnde des Halters dient hierbei der Sonde als Anschlag zur Begrenzung der Stichtiofe. Gegenüber dem bisher bei Sonden neist verwendeten, aus einem lose auf der Sonde verschiebbaren Scheibe aus Gumni oder dergleichen bestehenden Anschlag wird der Vorteil erzielt, daß ein Verschieben des Anschlages, wie dies bei den Scheiben oft vorkam, ausgeschlossen ist. Die Führung der Sonde s in dem Halter

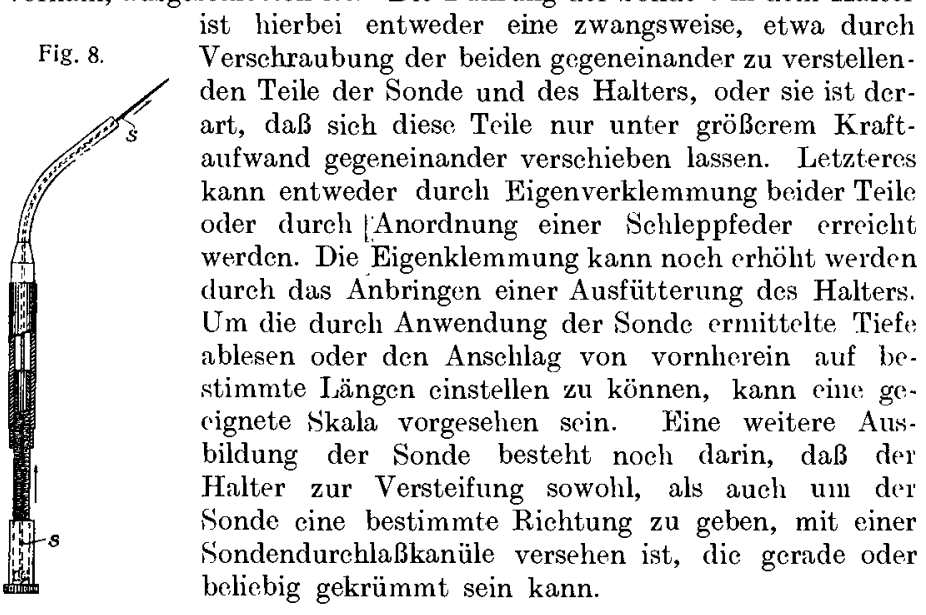

Un ein Zalmmesser mit einer von einem Stiel getragenen, zur Anfnalıme der Drahtschleife dienenden Platte handelt es sich bei D. R. P. 225505 (Dr. A. D. Cloud in New York); zwccks Frleichterung

Fig. 9.

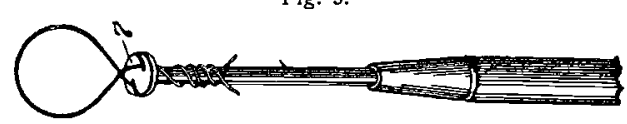

des Einfädelns der Drahtenden ist die Platte 7 vgl. Fig. 9) 1nit einander gegenüberliegenden geschweiften oder geraden Schlitzen versehen, dic sich von der Kante nach der Mitte erstrecken.

D. R. P. 225506 bezieht sich auf die besondere Ausbildung des Mundstïckes eines namentlich in der zahnärztlichen Praxis Verwendung findenden Speichelziehers (M. W. Levkowicż in San Francisco). An das starre Kniestïck des Mundstückes ist dreh- und gleitbar ein Rohrglied angeschlossen, das an seinem unteren Ende die bekannte Saugkappe trägt. Das Rohrglied ist aus einem winkligen, in das Kniestück geschobenen Teil und cinem geraden, dic Saugkappe 7 tragenden und auf den winkligen Teil aufgeschobenen Teil zusammengesetzt, welche beide gegeneinander und gegen das Kniestück gleitbar verstcllt und verdreht werden können (vgl. Fig. 10). Das Mundstück kann daher leicht den verschiedensten Mundhöhlen angepaßt werden, ohne cinen

Druck auf dic Zunge des Patienten auszü̈ben, und ist ferner leicht $\mathrm{zu}$ reinigen.

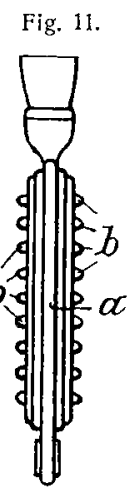

D. R. P. 225407 betrifft eine Wärmeflasche oler -geläß fiir Kompressen (Dr. A. Ritter von Borosini in Pillnitz bei Dresden). Die Außenfläche der Wärmflaschen- oder Gefäl3wandung $a$ ist nit zahlreichen wärmcisolierenden Vorsprüngen oder Erhöhungen b versehen (vgl. Fig. 11), welche die heißc Gefäßwand unter Bildung einer Luftschicht von der zu bchandelnden Körperstelle entfernt halten. Die Vorsprüngc oder Erhöhungen sind an einem über die Warmflasche oder das Gefäß zu ziehenden Ueberzug angeordnet. Infolgedessen kann eine solche Kompresse selbst naeb Füllung mit kochendem Wasser oder Salzlösungen von der zu behandelnden Körperstelle ertragen werden, "ohne daß eine Verbrennung der betreffenden Stielle zu befürchten ist.

Eine Menstruationshose ist durch D. R. P. 225409 geschützt (Osw. Rauft in Frankfurt a. M.). An die obere Kante des im ausgebreiteten Zustande völlig flach liegenden, wasserdichten Stoffes ist ein anf den Leib sich legender Oberteil aus Wärme isolierenden Stoff lösbar angesehlossen.

D. R. P. 224009 bchandelt cinen elastischen Saug. napf für Massagezwecke (W. Rittberger in Berlin). Bei den bisherigen Massagegeräten diescr Art war num die Führung des halbkugeligen Napfes unvollkommen, weil sich die Ränder des Napfes leicht umlegten und abhoben. Zur Behebung dieses Mangels ist gemäß vorliegender Erfindung zwischen dem Saugnapf a (vgl. Fig. 12) und dessen (trift f eine Fiihrungskapsel d verseliebbar angeordnet. (Schlnis folgt.)

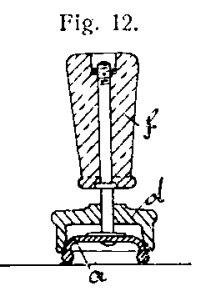

